\title{
Forensic Applications of Microbiomics: A Review
}

\begin{abstract}
Jake M. Robinson 1,2, Zohar Pasternak ${ }^{3}$, Christopher E. Mason ${ }^{4,5,6}$ and Eran Elhaik ${ }^{7 *}$
${ }^{1}$ Department of Landscape, University of Sheffield, Sheffield, United Kingdom, ${ }^{2}$ Healthy Urban Microbiome Initiative (HUMI), Adelaide, SA, Australia, ${ }^{3}$ Quality Assurance and Evidence Unit, Division of Identification and Forensic Science (DIFS), National Headquarters of the Israel Police, Jerusalem, Israel, ${ }^{4}$ Department of Physiology and Biophysics, Weill Cornell Medicine, New York, NY, United States, ${ }^{5}$ The HRH Prince Alwaleed Bin Talal Bin Abdulaziz Alsaud Institute for Computational Biomedicine, Weill Cornell Medicine, New York, NY, United States, ${ }^{6}$ The WorldQuant Initiative for Quantitative Prediction, Weill Cornell Medicine, New York, NY, United States, ${ }^{7}$ Department of Biology, Lund University, Lund, Sweden
\end{abstract}

The rise of microbiomics and metagenomics has been driven by advances in genomic sequencing technology, improved microbial sampling methods, and fast-evolving approaches in bioinformatics. Humans are a host to diverse microbial communities in and on their bodies, which continuously interact with and alter the surrounding environments. Since information relating to these interactions can be extracted by analyzing human and environmental microbial profiles, they have the potential to be relevant to forensics. In this review, we analyzed over 100 papers describing forensic microbiome applications with emphasis on geolocation, personal identification, trace evidence, manner and cause of death, and inference of the postmortem interval (PMI). We found that although the field is in its infancy, utilizing microbiome and metagenome signatures has the potential to enhance the forensic toolkit. However, many of the studies suffer from limited sample sizes and model accuracies, and unrealistic environmental settings, leaving the full potential of microbiomics to forensics unexplored. It is unlikely that the information that can currently be elucidated from microbiomics can be used by law enforcement. Nonetheless, the research to overcome these challenges is ongoing, and it is foreseeable that microbiome-based evidence could contribute to forensic investigations in the future.

Keywords: microbiome, forensic microbiology, forensic science, microbial forensics, metagenomics, postmortem interval, microbiomics

\section{INTRODUCTION}

For over 100 years, microbiology has played a relatively diminutive role in forensic science (MacCallum and Hastings, 1899). In the early 1990s, the sequencing of amplified viral DNA was used to support a case alleging the transmission of Human Immunodeficiency Virus from a dentist to several patients in Florida, United States (Smith and Waterman, 1992). The emergence of PCR-mediated genotyping of bacteria was considered to be a valuable forthcoming tool in forensics-e.g., van Belkum (1994) suggested that forensic science would soon be a major area for the application of PCR-mediated genotyping due to the rapidity of technological advances

Abbreviations: PCR, polymerase chain reaction; MetaSUB, metagenomics and metadesign of subways and urban biomes; PRISMA, preferred reporting items for systematic reviews and meta-analyses; CAMDA, critical assessment of massive data analysis; PMI, postmortem interval; OUT, operational taxonomic unit; CRP, C-reactive protein; SIDS, sudden infant death syndrome. 
at the time (van Belkum, 1994). In the mid-1990s, fungal and pollen spore analyses were also developed, allowing investigators to differentiate between soil types, which in turn allowed linking substrate items to particular sites (Bruce and Dettmann, 1996; Bryant and Mildenhall, 1998). However, it was not until the early 2000s and the rise of bioterrorism that microbial forensicsthe "scientific discipline dedicated to analyzing evidence from a bioterrorism act, biocrime, or inadvertent microorganism/toxin release for attribution purposes"-emerged in response to the new threat (Budowle et al., 2003; Carter et al., 2017).

Many forensic applications have been limited to individual taxa analyses, and microbial forensics has, historically, been constrained by a lack of available and cost-effective sequencing technologies (Berglund et al., 2011; Kuiper, 2016). This approach has changed dramatically in the last decade as advances in genomic sequencing technology, and new methods for processing complex community datasets (and often low biomass samples) have led to the advent of a new field of microbiomics. The science and study of the microbiome (Statnikov et al., 2013; Capasso et al., 2019) combined with metagenomics (all genomes from a sample) have enhanced the development of the microbial forensic toolkit (Clarke et al., 2017; Hampton-Marcell et al., 2017).

As of March 2019, the conviction rate for homicides in England and Wales (United Kingdom) was only 79\% (Office for National Statistics, 2019), slightly higher than in the US ( $70 \%$ ) (Bureau for Justice Statistics., 2019). Across the globe, there is also a high prevalence of wrongful convictions and often insufficient evidence to convict a perpetrator of a crime (Sangero and Halpert, 2007; Ingemann-Hansen et al., 2008; LaPorte, 2017; Walsh et al., 2017). According to the Innocence Project, a national litigation and public policy organization dedicated to exonerating wrongfully convicted individuals, to date, 375 people in the United States have been exonerated by DNA testing, including 21 who served time on death row (Innocent Project, 2020). There is thereby a strong interest from the public, lawmakers, and the law enforcement system to augment and expand the forensic toolkit, including molecular methods. Microorganisms are abundant in and on the human body (microbial cells can outnumber or equal the total number of human somatic cells) (Noel et al., 2014; Sender et al., 2016; Vázquez-Baeza et al., 2018), in surrounding environments, and on objects associated with a crime (Desmond et al., 2018; Oliveira and Amorim, 2018). A growing body of evidence suggests that forensically relevant microbial profiles could be used as evidence or, at the very least, complement traditional investigative methods (Metcalf et al., 2017; Schmedes et al., 2017; Richardson et al., 2019; Phan et al., 2020). This use of microbial profiles as evidence is done using computational tools that are being developed alongside new approaches in bioinformatics, processing tools, and refined protocols. However, since the field is still in its infancy (Goudarzi et al., 2016; Komaroff, 2018) and historically underfunded (Morgan and Levin, 2019), there is much uncertainty as to the true potential of microbiomic tools in forensics.

In this review, we provide an overview of past, current, and future potential applications of microbiomics in forensics. Specifically, we will discuss the six most comprehensively researched themes (Figure 1): including geolocation (e.g., substrate analysis and different spatial dimensions and the power of machine learning), personal identification, biological sex determination, trace evidence, manner and cause of death (e.g., death by drowning), PMI, and other applications (e.g., localization through animal microbiomes).

\section{MAJOR THEMES IN THE FORENSIC MICROBIOLOGY LITERATURE}

\section{Geolocation}

In the past few years, intensive work has been carried out to characterize environmental microbiome, particularly in urban environments and transit systems. These studies have demonstrated that unique community profiles may exist in certain areas of a city (Afshinnekoo et al., 2015; Rosenfeld et al., 2016), as well as "molecular echoes" of environmental events, and even a forensic capacity for geospatial microbiomic data (MetaSUB International Consortium, 2016; Danko et al., 2019a). In the following, we focus on two leading aspects of geolocalization.

\section{Substrate Analysis}

The potential of analyzing microbial profiles from the soil is increasingly being recognized in forensic microbiological research. Both the rhizosphere and bulk soil microbiomes exhibit a high level of heterogeneity between different sites. As such, with methodological refinement, soil microbiome samples could provide valuable biogeographic data to localize the origin of the soil sample. Another potential application is the acquisition of information to help determine the provenance of an item(s) associated with a crime.

Habtom et al. (2019) demonstrated distance-decay relationships between microbial samples from the soil at a local scale (25-1,000 m) ( $n=5$ sites, $n=2-4$ soil types, and five replications). The results showed that the greater the distance between the samples, the more they differed, suggesting that both soil type and geographic location are important factors in determining microbial community composition. Indeed, patch discrimination using the soil microbiome has previously been demonstrated (Macdonald et al., 2011), and Jesmok et al. (2016) correctly classified $95.4 \%$ of soil bacterial profiles to their location of origin using various methods including abundance charts, non-metric multidimensional scaling, analysis of similarity, and $k$-nearest neighbor. However, this was a feasibility study with a modest sample size $(n=19)$. Further studies with larger sample sizes and replications are needed to explore the full potential of this approach.

Evaluating the microbial communities in an assemblage of soil samples (e.g., soils from a crime scene or alibi site and other intermediary sites) could be useful in forensics. Samples originating from a mixture of different soil substrates have been correctly differentiated by using a combination of Ribosomal Intergenic Spacer Analysis and 16S rRNA gene sequencing (Demanèche et al., 2017). Recent evidence suggests that $18 \mathrm{~S}$ rRNA gene sequencing can also provide greater discriminatory power over traditional Mid-Infrared spectroscopy at fine scales 


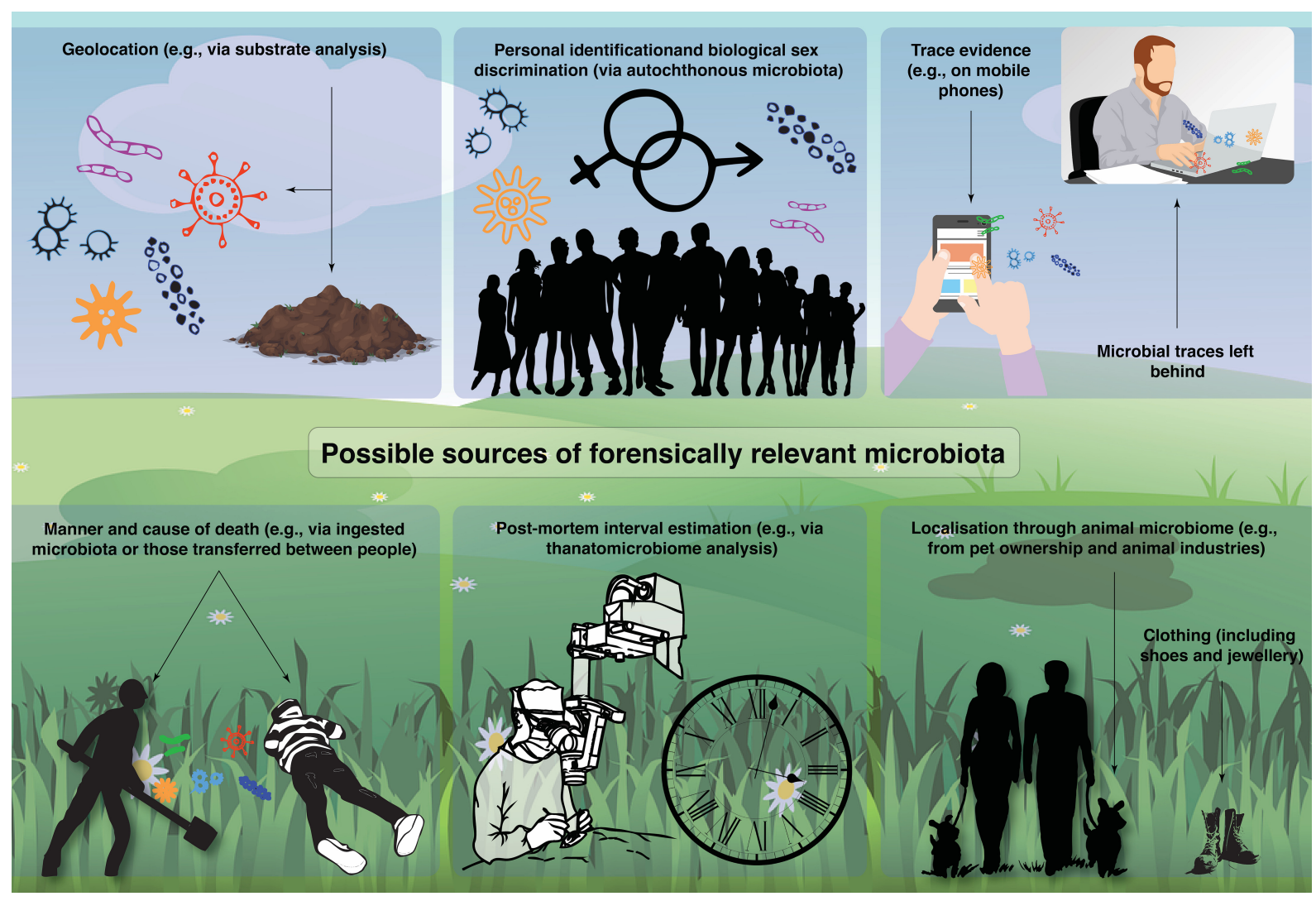

FIGURE 1 | A summary of possible sources of forensically relevant microbiota identified by the literature review.

for eukaryotic species (Young et al., 2015). Furthermore, Sanachai et al. (2016) demonstrated that the site origin of soil, obtained from the sole of a shoe, could be elucidated by comparing the similarity of soil bacterial $16 \mathrm{~S}$ rDNA profiles acquired by the denaturing gradient gel electrophoresis technique.

Despite the potential in this field, further limitations need to be identified and addressed. For example, Pasternak et al. (2019) identified several potentially limiting factors to consider when interpreting the results of microbiomic analyses. For instance, soil samples are incredibly complex and highly heterogeneous even at short spatial scales, which presents a major issue to using these in a forensic context, and microbiomes exhibit a high level of physical, chemical, and biological diversity in both space and time. Pasternak et al. (2013) showed that actinobacterial fingerprints significantly differed between two seasons (summer and winter) at the same sites-implying that temporally-associated issues could arise. Keet et al. (2019) have also pointed out that soil microbiome composition can change as a result of abiotic soil conditions and plant community patterns-which poses a considerable challenge to the accuracy of results.

Metagenomic analysis of gravesoil and the soil around human and non-human animal cadavers has been undertaken for forensic purposes. Carter et al. (2015) investigated microbial community succession in soils associated with swine cadavers across two seasons (summer and winter). They demonstrated that postmortem microbial communities changed in specific and reproducible ways, but decomposition effects on soil microbial communities differed significantly between seasons. The authors suggest that the ecological succession of microbial communities will be useful for forensic investigations, but future research should aim to gain a greater understanding of seasonality on decomposition. The sample size for this study was not explicitly stated, but according to the ordination plots, it appears to be modest ( $n \leq 10$ per treatment). Therefore, results should be interpreted with caution.

Adserias-Garriga et al. (2017) investigated daily thanatomicrobiome changes in the soil as an approach to estimate PMI. They collected soil samples from around human cadavers ( $n=1$ male and $n=2$ females) and demonstrated successional changes on a daily basis. Rapid growth of Firmicutes was observed from the bloat stage to advance decay $(<5 \%$ relative abundance at day 1 to $75 \%$ relative abundance day 12), and the authors proposed a Firmicutes growth curve to estimate PMI. However, the authors state that the growth curve results may only apply under Tennessee summer conditions and that confirmatory research is needed using a larger number of cadavers and under different environmental conditions. The results do, however, corroborate those of Finley et al. (2016), who evaluated microbial communities associated with 
gravesoil human cadavers $(n=18)$. The researchers allowed the cadavers to decompose over a range of decomposition time periods (3-303 days) and showed increases in the relative abundance of Firmicutes in surface bodies over the decomposition period (from $\sim 10 \%$ at $0-3$ months to $\sim 40 \%$ at 7-9 months).

Singh et al. (2018) investigated the spatial (0,1, and $5 \mathrm{~m}$ ) dynamics of human cadaver decomposition on soil bacterial community structure. They collected soil samples from each spatial buffer ( $n=14$ for the $0 \mathrm{~m}, n=17$ for both 1 and $5 \mathrm{~m}$ ) and observed evidence of a predictable response to cadaver decomposition that varied over space. Bacterial community composition (beta diversity) at $0 \mathrm{~m}$ was significantly different from the 1 and $5 \mathrm{~m}$ communities, whereas there were no significant differences between the 1 and $5 \mathrm{~m}$ communities. The researchers also found that bacterial alpha diversity was significantly lower in the $0 \mathrm{~m}$ samples, suggesting that the additional nutrient input from the cadavers may reduce bacterial alpha diversity. This study provides additional spatialcompositional insights to complement the growing body of knowledge in this area of forensic microbiome applications.

Soil microbiome analysis has the potential to be used in forensics; however, additional research is required to validate the sensitivity and reproducibility of results (Young et al., 2017). Overall, to gain a greater understanding of both spatial and temporal dynamics associated with the microbiome and to develop techniques to mitigate similar pitfalls, further microbiome surveys are essential.

\section{Different Spatial Dimensions and the Power of Machine Learning}

The growing interest in sampling and predicting environmental microbiome profiles at different spatial scales and orientations (e.g., between households, cities, states, and altitudes) to provide information on the location and provenance of people and objects resulted in the development of a multitude of approaches. Chase et al. (2016) identified the three cities where nine offices were located with $85 \%$ accuracy based on analyzing office microbiome samples using sampling plates, and although this study suffers from a small sample size ( $n=3$ per office), it demonstrates potential with further refinement. Lax et al. (2014) analyzed samples from household occupants $(n=1625$ from 18 participants in 10 houses) and their built environments. The authors matched feet microbiome samples to the house with $82.9 \%$ accuracy - a relatively low degree of accuracy from an evidentiary perspective but demonstrating the potential of such methods for fine-scale biolocalization. Walker and Datta (2019) analyzed whole-genome sequenced microbiota sampled from 12 cities in seven different countries as part of the 2018 CAMDA MetaSUB Forensic Challenge. The CAMDA dataset $(n=30)$ included three mystery samples. The authors applied machine learning techniques to identify the geographical provenance of the microbiome samples. Up to $90 \%$ of the samples were correctly classified, demonstrating the potential of machine learning applications to biogeography, although further evidence is necessary to employ these applications in an evidentiary context. In a related study, Ryan (2019) applied a random forest classifier built on a dataset of 311 city microbiome samples. Their method correctly classified $83.3 \%$ of the mystery samples. Grantham et al. (2019) presented a different algorithm for predicting the geolocation of fungal samples from dust $(n=1300)$ in the United States using deep neural network classifiers. Applied to a global dataset of samples from 28 countries, the authors state that their algorithms make "good point predictions" with $>50 \%$ of the geolocation errors under $100 \mathrm{~km}$ for US-wide analysis and nearly 90\% classification accuracy of a sample's country of origin for the global analysis. This particular field, combining microbiomics and machine learning, is in its infancy, and future studies would benefit from larger sample sizes and improved classification accuracy before such approaches can be used with confidence in a forensic context.

Another important spatial factor to consider is that microbiome compositions do not only differ in horizontal space. Skin microbiomes have also been shown to differ between humans living in high and low altitudes. For example, Zeng et al. (2017) collected skin microbiome samples from humans $(n=99)$ and pigs $(n=82)$ in Tibet. They found enrichments of several bacterial taxa (e.g., Arthrobacter sp., Paenibacillus sp., and Carnobacterium sp.) in samples collected from higher altitudes. Alpha diversity was also significantly lower in skin samples collected from individuals living at higher altitudes. This suggests a potential future route to determine geolocation based on altitudinal parameters via the analysis of skin microbiome samples in the future-although here too, methodological refinement will be essential. Furthermore, understanding how skin microbiomes may fluctuate throughout the life course will also be an essential factor to consider.

Overall, all the models prioritize classification over prediction abilities. To enable real-time prediction of geographical coordinates from sampling data, increasing the sample sizes geographically and temporally, and developing more rigorous methods is essential.

\section{Personal Identification}

A growing body of evidence suggests that human individuals may be uniquely identified based on stable autochthonous (i.e., native to a given environment) microbial profiles. This could have a substantial impact on forensic science-for example, in situations where the investigator cannot retrieve sufficient amounts of human DNA (i.e., from human somatic and germ cells). Yet it is unknown whether the variation in microbial communities between people is sufficient to identify individuals within large populations uniquely or stable enough to place them over time.

To answer some of these questions, Franzosa et al. (2015) tested different body site-specific microbial profiles and attempted to match them with 25-105 microbiome profiles during the person's first and second visits to the sampling site. The authors reported that these profiles were useful in distinguishing individuals at the initial sampling time point and that $30 \%$ of the individuals were still uniquely identified several months later. In this study, gut microbiome samples were used to pinpoint $80 \%$ of individuals $(n=120)$ up to 
a year later. These results are encouraging-particularly in shorter timescales-however, they still suffer from relatively high variability. As such, greater improvements, e.g., in methods and sampling effort, will be needed before such approaches can be useful in a forensic setting.

High resolution melting analysis that targeted the $16 \mathrm{~S}$ rRNA gene from oral swab samples have also been used to demonstrate its potential in distinguishing between individuals (Wang et al., 2019), albeit with a very small sample size in this study $(n=5)$. Schmedes et al. (2018) demonstrated accurate identification of individuals $(n=12)$ based on skin swab samples from different body sites $(n=14)$. They achieved $97 \%$ accuracy by sampling shirts and $96 \%$ accuracy using palm samples based on 1-nearest neighbor classification on nucleotide diversity of the bacterial genome. In another recent study, the researchers utilized a similar approach to identify individuals $(n=51)$. They analyzed microbiome samples collected from three different body sitesthe manubrium (i.e., the upper-most segment of the sternum), the palmar surface of the hand, and the ball of the foot (Woerner et al., 2019). The researchers achieved 100\% classification accuracy when conditioned on a maximum nearest neighbor distance for diversity, suggesting strong potential should these results be replicable in studies with much larger sample sizes.

Watanabe et al. (2018) suggested that minor taxa are one of the key factors for distinguishing between individuals. Their study analyzed microbiome samples $(n=66)$ from individuals $(n=11)$ over 2 years and achieved $85 \%$ accuracy in distinguishing individuals. They also used the same analytical methods to classify publicly available skin microbiome samples from individuals $(n=89)$ with a $78 \%$ identification accuracy. However, this level of accuracy is unlikely to be sufficient for forensic applications. The authors suggested that although personal identification is possible, the estimation of the accuracy decreases for larger cohorts due to increments of similar microbiome patterns. Overall, the use of microbiomics as a forensic tool to determine personal identification shows potential and technological viability and might be useful in situations where the investigator is unable to retrieve sufficient amounts of human DNA. Nonetheless, the findings fall short of the burden of proof. Improvements in the model's sensitivity and specificity are required, and a methodology to address potential contamination issues. Furthermore, a better understanding of the microbial dynamics across time and space is essential for the findings to have a forensics value.

\section{Biological Sex Determination}

Recent evidence supports another contribution of microbiomics toward personal identification -the discrimination of biological sex, which could be useful where sufficient quantities of human DNA are unable to be retrieved. For example, airborne bacteria communities have previously been characterized in indoor environments (Chan et al., 2009). Luongo et al. (2017) investigated airborne bacterial and fungal diversity (i.e., constituents of the "aerobiome") from different University dormitory rooms $(n=91)$. They used machine learning techniques and were able to predict the biological sex of room occupants with $79 \%$ accuracy based on relative abundances of the microbiota. Curiously, rooms occupied by males exhibited higher relative abundances of the microbiota. The authors suggested that it could be because males may shed more biological particles or use fewer cosmetic barriers such as skin lotions.

Biological sex-related differences in the human thanatomicrobiome-the microbial communities colonizing organs following death (thanatos, Greek for death) (Zhou and Bian, 2018)- have also been demonstrated by Bell et al. (2018). The authors compared amplicon signatures (using the 16S rRNA gene V1-2 and V4 regions) in the corpse heart tissue of 10 individuals and discovered key differences between males $(n=6)$ and females $(n=4)$. For example, Streptococcus sp. was found exclusively in male heart tissues, whereas females had a significantly higher prevalence of Pseudomonas sp. With refinement, such an approach could help to determine the biological sex of a corpse and the provenance of body parts.

In a study by Tridico et al. (2014), the authors "readily distinguished" male $(n=3)$ and female $(n=4)$ subjects based on the analysis of their pubic hair microbiomes. They identified Lactobacillus spp. that were unique to female participants. They also suggested that pubic hair is relatively insulated from the environment and colonized with niche-specific microbiota, which could be useful in forensic investigations. Unfortunately, the modest sample size of this study limits the conclusions that can be drawn from it. Nonetheless, the findings were supported by another small study by Williams and Gibson (2017), who identified individuals $(n=9)$ and their biological sex from pubic hair microbiota with an error ratio of $0.027 \pm 0.058$ and $0.017 \pm 0.052$, respectively. However, the sample sizes for all these studies are modest, and as such, further validation studies with larger sample sizes are needed before reliable conclusions can be drawn.

Interestingly, Phan et al. (2020) analyzed skin microbiome samples from both genders $(n=45)$ and found that the absence of the bacterial genus Alloiococcus could be useful in predicting female biological sex. The study showed a correlation between certain bacterial species and personal characteristics (e.g., biological sex). They specifically explored the presence/absence of microbiota from fingermarks left behind on surfaces and achieved a relatively modest $67 \%$ sex prediction accuracy using leave-one-out cross-validation analysis. Improvements in sample sizes and machine learning accuracy are necessary to explore the potential of this approach further. Additional research into whether certain bacteria (and other microorganisms) are distinct to females or simply related to external factors (such as cosmetic products on hands) would also be necessary.

\section{Trace Evidence}

There is an increasing interest in studying forensically relevant microbial profiles left behind on objects and surfaces. For instance, several studies showed that there is often a high level of bacterial presence on personal objects such as mobile phones (Koroglu et al., 2015; Kõljalg et al., 2017; Koscova et al., 2018; Kurli et al., 2018). Furthermore, human-associated items such as shoes and mobile phones have been shown to support distinct microbiomes (Lax et al., 2015; Coil et al., 2019). 
Meadow et al. (2014) investigated the potential utility of mobile phones as "personal microbiome sensors." They selected 17 individuals and collected three samples (the cell phone's touch surface, their index finger, and their thumb). They demonstrated that bacterial communities sampled from mobile phones were more similar to their owners than other people. They found that about $22 \%$ of the taxa on participants' fingers were also found on their phones, whereas only $17 \%$ were shared with other people's phones. An individual's index finger shared approximately 5\% more OTUs with their mobile phone than with everyone else's mobile phone in the study. Furthermore, $82 \%$ of the OTUs were shared between a person's index finger and their phone. Although promising, here again, the sample size and accuracy of results need to be increased in future studies.

Kodama et al. (2019) found that postmortem skin microbiomes could be associated with personal objects with a high degree of accuracy. Several of the objects in the study were associated with $100 \%$ accuracy (i.e., medical devices, eyeglasses, bottles, and steering wheels), whereas objects like computer devices, remote controls, and cell phones were associated with over $67 \%$ accuracy, suggesting that with refinement, skin microbiome samples could be reliably linked to objects at the scene. Furthermore, studies have found that the postmortem skin microbiomes were stable and similar to antemortem skin microbiomes for up to $60 \mathrm{~h}$ postmortem (Kodama et al., 2019).

Salzmann et al. (2019) investigated the microbial profiles of different bodily fluids $(n=22)$. They identified sourcespecific microbial signatures from various bodily fluids. For example, the phyla Proteobacteria was associated with skin and semen sources, whereas Firmicutes showed a higher prevalence in saliva and vaginal secretions. Dobay et al. (2019) suggest that even when body fluid is exposed to indoor conditions for 30 days, samples continue to harbor body-site-specific microbial signatures. Hanssen et al. (2017) also demonstrated promising results, albeit with a small sample size $(n=6)$, for the microbiallymediated classification of body fluids. They performed pattern recognition by fitting a linear discriminant analysis model using Principal Component scores and were able to classify saliva samples in $94 \%$ of the cases.

Neckovic et al. (2020) recently investigated the potential transfer of skin microbiomes between individuals and substrates (i.e., allochthonous microbiota). They found that skin microbiota has been reliably transferred through direct contact, that is, between individuals shaking hands. Microbiota also transmits through indirect contact, as demonstrated by individuals rubbing a substrate and then swapping substrates with another person. The authors suggested that such analysis could be useful to corroborate sexual assault cases or other contact-related crimes. They also suggested that further research should consider the relative surface area of contact, pressure, friction, and the duration of the contact.

\section{Manner and Cause of Death}

The 'manner of death' is a determination made by an expert following an investigation (e.g., a coroner, the police, or a medical examiner). Five manners of death are generally considered: natural, accidental, suicide, homicide, and undetermined
(Advenier et al., 2016). Lutz et al. (2019) recently collected microbiome samples from 265 corpses from Finland, Italy, and the United States. The inspected cadavers differed in the manners of death: accidental death $(n=88)$, natural death $(n=106)$, homicide $(n=23)$, and suicide $(n=45)$. Their results suggested that Lactobacillus, Enterobacteriaceae, Sediminibacterium, and Rhizobiales were associated with different manners of death. With further research, these associations could be developed into predictive markers that help to determine the manner of death. However, as noted by the authors, Sediminibacterium and Rhizobiales bacteria may also represent environmental contamination, which needs to be controlled, and further validation through controlled experiments is needed to improve the reliability of their approach to determine the manner of death.

The potential of this microbiomics approach to determining the manner of death was corroborated in a recent study by Zhang et al. (2019) who, by obtaining samples during routine death investigations at the Wayne County Medical Examiner's Office (Detroit, Michigan, United States), found different biomarkers associated with the manner of death. In this study, Xanthomonadaceae was more prevalent in cases related to hospital deaths, whereas Actinomyces sp. tended to be more prevalent in suicide cases. Increasing the numbers of samples generally increased the accuracy of the models. The authors cautioned that the prediction accuracy depends on the machine learning methods used and the number of anatomical sites analyzed. The authors suggest this study provides baseline information, and it could be possible to use machine learning to develop reference databases that allow microbially-mediated manner of death predictions in the future.

Kaszubinski et al. (2020) modeled beta-dispersion to test for manner and cause of death association using a microbiome data set of $n=188$ postmortem cases (five body sites per case). The researchers demonstrated that beta-dispersion and demographic data could distinguish among manner and cause of death. In particular, they found that cardiovascular disease and drugrelated deaths were correctly classified in $79 \%$ of cases. They found that binary logistic regression models were most effective at improving model success. This was an improvement over multinomial logistic regression models, which confirmed the manner and cause of death assessment only $62 \%$ of the time. The results of this study show promise for using postmortem microbiomes to indicate the manner of death. However, as the researchers' highlight, sample sizes need to be greater. Moreover, the development of large databases will likely be required to train models with high success rates prior to being used in practical forensic contexts.

In terms of cause of death (i.e., the disease or injury that produces physiological disruption in the body leading to death), researchers such as Christoffersen (2015) have investigated the importance of microbiological testing. Studying autopsy results $(n=42)$, the author reported that the cause of death could be determined in $42 \%$ of the cases via microbiological analysis. The study highlighted factors indicative of a microbiologically related cause of death, such as a raised CRP measurements. 
Raised CRPs have also been implicated in SIDS as a cause of death (Rambaud et al., 1999; Szydlowski et al., 2013) and even for astronauts returning from space (Garrett-Bakelman et al., 2019). Deadly bacterial infections, such as infection or sepsis, may also occur following neonatal circumcision (Elhaik, 2016, 2019).

A specific forensic microbiome application for determining the cause of death is the diagnosis of 'death by drowning, which is one of the leading causes of unnatural deaths worldwide (Domínguez et al., 2018; Cenderadewi et al., 2019). Analyzing the presence of diatoms (single-celled algae) has been the 'gold standard' for well over a decade; however, its reliability has been questioned (Kakizaki et al., 2009; Huys et al., 2012). Several studies have provided support for death by drowning diagnoses by designing real-time PCR assays with primers to detect bacterial species associated with aquatic environments, such as Aeromonas spp. (Aoyagi et al., 2009; Uchiyama et al., 2012; Rutty et al., 2015; Voloshynovych et al., 2019). These studies provided support for this cause of death diagnosis based on relatively high detection rates of microbiota, for example, $84 \%(n=32), 75 \%(n=20)$, and $84 \%(n=43)$-although to strengthen the cause of death diagnoses, the accuracy levels, and sample sizes could again be much improved. It has also been suggested that bioluminescent bacteria may be biomarkers for death by drowning in seawater. For example, Kakizaki et al. (2009) developed a simple assay targeting the 16S rRNA gene to identify bioluminescent colonies such as Vibrio fischeri and Vibrio harveyi. More recently, Lee et al. (2017) analyzed microbiome composition and pulmonary surfactant protein (SPA) expression to develop a marker for diagnosis of death by drowning. They analyzed microbiota and histological appearance of both drowned and postmortem groups of experimental rats, comparing freshwater vs. marine water treatments. The authors found that 5513 and 5480 OTUs were unique to marine and freshwater, respectively. They also found that expression levels of SP-A were higher in the lungs of drowning victims compared to postmortem submersion. These findings could have important forensic value (e.g., determining both the type of environment and the timing of death) and demonstrate good potential for future applications. Marella et al. (2019) point out that other studies have focused on the presence of fecal bacteria, coliforms, and streptococcal bacteria to help determine the cause of death by drowning. These bacteria are sampled from the femoral artery and vein and the right and left ventricles. Fecal bacteria are considered to be always present in subjects who drowned compared with those with other cause of death diagnoses (Lucci et al., 2008; Marella et al., 2019). For example, Lucci and Cirnelli (2007) found fecal streptococcal presence in $100 \%$ of the freshwater drowning cases they studied $(n=22)$ and coliforms present in $90.91 \%$. In this study, the control subjects $(n=30)$ uniformly showed an absence of fecal bacteria. In a later study, Lucci et al. (2008) assessed if the presence of these bacteria in the drowning medium could be detected in victims submerged after death. The researchers collected samples from drowned victims $(n=5$ freshwater and $n=5$ in seawater) and victims who were submerged after death $(n=3)$. Coliforms and streptococci were detected in all drowned victims but not in those submerged after death. These findings suggest that fecal coliforms and streptococci could be used as markers of drowning. However, the minuscule sample sizes must be interpreted with caution and increased considerably in future studies.

\section{Postmortem Interval The Thanatomicrobiome}

Determining the PMI (the time elapsed since a person has died) is often an essential part of a criminal investigation. To improve PMI prediction accuracy, researchers have begun examining the thanatomicrobiome (Javan et al., 2016; Burcham et al., 2019). Postmortem, these communities overwhelm the immune system allowing for subsequent colonization (Javan et al., 2019). Preliminary studies suggest that these microbial communities may undergo important successional changes in organs that could aid in determining the PMI (Adserias-Garriga et al., 2017).

Early studies on model animals suggest that this is feasible. For over a 48-day period of decomposition, Metcalf et al. (2013) aimed to uncover a "microbial clock" to provide an estimate of PMI by sequencing the 16S rRNA gene for bacterial and archaeal communities and the 18S rRNA gene for microeukaryotes. Their model provided reliable PMI estimates ( \pm 3 days) ( $n=223)$. However, the study was conducted in controlled conditions using experimental mouse modelsthereby necessitating a degree of caution when extrapolating the data to 'real-life' situations. Another study investigated the decomposition of pig cadavers. Their model predicted the PMI within 2-3 h of the time of death with 94.4\% accuracy (Pechal et al., 2014), demonstrating promise with further methodological refinement. Pechal et al. (2018) carried out a large-scale study of body microbiome samples $(n=188)$ that found postmortem microbiomes were stable, reflecting antemortem microbiomes 24-48 $\mathrm{h}$ after death. The researchers also found that specific bacterial taxa were important in predicting health status. For example, Haemophilus and Fusobacterium were twice as abundant in healthy individuals, whereas Rothia was 0.09 times more abundant in heart disease cases. With further development, this could be used to indicate the state of human health during clinical investigations into a range of deaths, from chronic and natural to sudden and violent (Pechal et al., 2018). It is important to note that, although appropriate at the time, the bioinformatics approach used to process OTUs and to make functional predictions (e.g., QIIME 1.8 and PICRUSt 1 ) is now considered to be outdated. Furthermore, Amplicon Sequence Variants (ASV) may provide a richer taxonomic picture (Callahan et al., 2017).

Studying human subjects, Johnson et al. (2016) sampled the skin microbiome of decomposing human cadavers and developed an algorithm to estimate PMI. The authors achieved low error rates for skins samples and a PMI estimation accuracy of \pm 2 days ( $n=144$ from 21 cadavers), a substantial improvement compared to prior efforts (e.g., via entomological analysis). Belk et al. (2018) used 16S rRNA amplicon sequencing and found that creating models with the class or phylum taxonomic levels provided the most accurate predictions of PMI. This finding corroborated 
the study by Johnson et al. (2016) and illustrated its potential usefulness for forensics.

Another study using 454 pyrosequencing to determine abundances and diversity of the postmortem microbiome in several key organs such as the brain, heart, liver, and spleen found varying PMIs ranging from 29.5 to $240 \mathrm{~h}$ (Can et al., 2014). This study revealed that the most abundant taxa in postmortem microbial communities were the anaerobic, sporeforming Firmicute bacteria, Clostridium sp. Javan et al. (2017) confirmed that Clostridium sp. dominated at long PMIs, adding evidence to support the use of microbiomics in PMI determination in the future.

\section{Localization Through Animal Microbiomes}

Several studies have shown that animals from different taxonomic groups and environments possess unique microbial profiles. For example, Tibetan chickens Gallus gallus, Chinese Rhesus macaques Macaca mulatta, and plateau sheep Ovis spp. have unique gut microbiomes (Zhou et al., 2016; Huang et al., 2017; Zhao et al., 2018) shaped by genetic, geographical, and altitudinal factors. It has been demonstrated that the skin microbiomes of Estrildid finches, amphibians, bats, cetaceans, and dogs Canis lupus familiaris are unique (McKenzie et al., 2012; Avena et al., 2016; Erwin et al., 2017; Torres et al., 2017; Engel et al., 2018; Russo et al., 2018). Interestingly, Song et al. (2013) found that humans share microbial communities with their dogs.

With further investigations and methodological refinement, such capabilities point to the potential feasibility of linking a person with a site based on shared microorganisms with animals. Although further studies are needed, there is potential for forensic pathways to associate trace microbial profiles obtained from other species (unique to the given species) to a given environment and/or occupation (e.g., animal industries) or to pet ownership. For example, non-human animal-specific microbiota could potentially be detected on the body or clothing of a suspect or victim, which may be useful in the absence of sufficient animal DNA (i.e., from somatic and germ cells) evidence. This profile could then conceivably be traced to the point of contact with an animal or animal-based environments such as equine stables, pet shops, or zoos, thus complementing other traditional forensic evidence. However, this approach is mostly theoretical at the moment, and future research will be needed to test its feasibility.

\section{DISCUSSION}

As of today, microbiome-based forensics are almost absent from criminal investigations and courts. To explain why this is so, we may divide the different possible applications of microbial forensics into two groups: first, reconstruction issues such as the cause of death and PMI, which ask "what happened?" and help elucidate the circumstances of the crime; and second, comparison issues such as geolocation and personal identification, which ask "how similar are these two DNA profiles?" and may (dis)connect a suspect from an object or a place (e.g., murder weapon or crime scene). Reconstruction applications for forensic use are easier to develop since competing propositions are usually welldefined and limited in number; if sufficient research is invested in ascertaining the microbial characteristics associated with each combination of possible environmental, spatial and temporal conditions, then reconstruction becomes straight-forward. For example, if a cadaver is found buried at a depth of $1 \mathrm{~m}$ in a desert in summertime, and the temporal succession of the gut microbial community for these environmental conditions has previously been established, then PMI can be inferred with a high degree of accuracy and certainty. Thus, reconstruction microbiomics can readily pass the Daubert standard set by the US supreme court (Daubert v. Merrell Dow Pharmaceuticals Inc, 1993) to be recognized as admissible evidence bearing sufficient scientific foundation, including general acceptance in the scientific community, known and acceptable rate of error, and so on.

Comparison microbiomic tools, on the other hand, may provide greater benefit to the criminal investigation but are harder to develop to a level that would satisfy the Daubert standard. The most beneficial way to employ such tools would be in a "one-to-many" configuration, similar to forensic human DNA analysis: a DNA profile from trace evidence is compared to all the profiles (from known persons and locations) in a database, and if it exists in the database, its frequency in the relevant population (e.g., of soils) is calculated to enumerate the probability of encountering this profile by chance (i.e., originating from a location or person unrelated to the crime). At this time, however, there are hardly any relevant forensic databases of microbiomes that can be compared to trace evidence. In their absence, the only way to proceed in a forensic context is in a "one-to-one" configuration. For each criminal case, questioned samples (e.g., from a suspect's shoe) are compared to context samples from the crime scene, alibi area, and other relevant sites. This approach provides less benefit to the investigation because it can only give conclusions of exclusion, that two samples do not share the same origin, or relative conclusions, such as "sample A is more similar to sample B than it is to sample C." Inclusionary conclusions such as "sample A is very similar to sample B; the probability of a different sample, from another place, person or time, being this similar to sample $\mathrm{B}$ at random is 1 in X Million" is impossible without either an extensive database (which is costly to build and maintain) or thorough theoretical knowledge (which we do not have yet) regarding the factors that shape microbiomes. However, even for "one-to-one" analyses, we can provide a statistical evaluation of the evidence (Habtom et al., 2019) based on the Likelihood Ratio framework as recommended by the European Network of Forensic Science Institutes (Willis et al., 2015).

There are three more major hurdles to forensic tools of comparative microbiomics. The first one involves samples of mixed origins, containing substrates or DNA from different locations or persons. DNA analysis of mixtures is difficult even with simple human DNA profiles, and certainly more so with microbiomic DNA profiles which are far more complex. It is often impossible to tell with certainty which, or even how many, disparate microbiomes are present in the mixture, let alone accurately infer the DNA profile of each one. The famed 2016 
report of the US President's Council of Advisors on Science and Technology [President's Council of Advisors on Science and Technology (US), 2016] found that the prevalent subjective analysis of complex human DNA mixtures by forensic experts is not a reliable methodology. Consequently, several computer programs were developed to interpret complex human DNA mixtures in an objective manner, and these are slowly being validated and accepted for routine forensic use. Software for objective analysis of microbiomic DNA mixtures may be built on this basis, but these are still years in the future. The second major hurdle is temporal variation. Contrary to human DNA, which can remain unchanged for years, microbial communities (both on the body and deposited as trace evidence) can fluctuate over time, often in correlation with changes in environmental parameters like moisture and pH (e.g., Pasternak et al., 2013). In cases where two samples for comparison are obtained at different times when markedly different environmental conditions prevail, mitigating the temporal changes in community structure is needed before analysis can ensue. So far, only a few studies have addressed this topic experimentally, mainly by applying various carbon sources to force the different microbial communities to "converge" (Pasternak et al., 2019), but so far with limited success. The third, and perhaps most challenging hurdle, is the problem of DNA transfer. In the past decade, human DNA evidence gained widespread credibility and acceptance in the courts so that the identification of a DNA profile from trace evidence as originating from a specific person is rarely disputed nowadays. Instead, it is becoming more and more common for the defense to challenge the method of deposition of the DNA, suggesting that it reached the crime scene by a legitimate activity (before or after the crime occurred) or by DNA transfer (e.g., when the innocent suspect shook the hand of the real perpetrator). This hurdle can sometimes be overcome by using the likelihood-ratio approach with activity-level propositions (Mayuoni-Kirshenbaum et al., 2020); however, similar to the former hurdles, this one is also very much still an open question, and it will take more time, effort, and research before microbiomics is ready to be employed and accepted within the legal system.

\section{CONCLUSION}

Over the last decade, advances in genomic sequencing and bioinformatics have given rise to microbiomics, which fructified in a growing compendium of tools seeking to explore the panoply of microorganisms present in our bodies and environment. The evidence examined in this review indicates that microbiomics could be a forensically relevant and promising discipline with a multitude of applications-from determining substrate provenance and acquiring trace evidence to identifying individuals and estimating PMI. These advances may allow various microbiomic data, like those obtained from thanatomicrobiome analysis, to be used by forensic scientists to address questions related to criminal investigations, or at least be used alongside other forensic methods.

Throughout their life-course, humans and their microbiomes undergo complex interactions and co-adaptation processes involving nutrient intake and resulting in the production of decomposition products such as metabolites. Following a person's death, these interactions change dramatically, and the microbiome composition and dynamics fluctuate accordingly. Understanding these colonizations and fluctuations represent major conceptual, methodological, and computational challenges-as do antemortem microbial dynamics. Related microbiome-based research in a forensics context and greater exploration of fungal and viral communities may also lead to an important enhancement in the forensic toolkit in the future.

Many challenges remain to overcome, such as contamination issues, modest study sample sizes, model over-specification and misspecification, prediction accuracies of machine learning techniques, understanding complex spatial and temporal variations in environmental microbiome dynamics, as well as risks and ethical concerns (Shamarina et al., 2017). Notably, even human DNA-based evidence, which is far better understood, is not error-proof, as indicated by the Phantom of Heilbronn case (Daniel and van Oorschot, 2011). Moreover, the vast majority of published work used $16 \mathrm{~S}$ or targeted sequencing approaches, which have known limitation for taxonomic resolution and could likely benefit from metagenomics methods (Ogilvie and Jones, 2015; McIntyre et al., 2017) and/or methods that utilize longer reads (Danko et al., 2019b; Foox et al., 2020). Also, more field-based testing and deployment of these sequencing methods could benefit from rigorous, titrated standards for ensuring accuracy (McIntyre et al., 2019). Given this, many of the applications reported in the literature should be considered proof of concepts rather than full-fledged forensic applications. Nonetheless, as Ogilvie and Jones (2015, p. 1) have summarized: "it is clear that we remain in a period of discovery and revelation, as new methods and technologies begin to provide [a] deeper understanding of the inherent ecological characteristics of this [microbial] ecosystem."

\section{AUTHOR CONTRIBUTIONS}

EE initiated the study. JR carried out the review. EE, JR, ZP, and $\mathrm{CM}$ wrote the manuscript. All authors approved the manuscript.

\section{FUNDING}

Payment for open access publication was made by Lund University. JR was undertaking a Ph.D. through the White Rose Doctoral Training Partnership (WRDTP), funded by the Economic and Social Research Council (ESRC). Grant code: ES/J500215/1.

\section{ACKNOWLEDGMENTS}

We would like to thank the Starr Cancer Consortium (I130052), the Vallee Foundation, the WorldQuant Foundation, The Pershing Square Sohn Cancer Research Alliance, and the National Institutes of Health (R01AI151059), the NSF (1840275), and the Alfred P. Sloan Foundation (G-2015-13964). 


\section{REFERENCES}

Adserias-Garriga, J., Hernández, M., Quijada, N. M., Lázaro, D. R., Steadman, D., and Garcia-Gil, J. (2017). Daily thanatomicrobiome changes in soil as an approach of postmortem interval estimation: an ecological perspective. Forensic Sci. Int. 278, 388-395. doi: 10.1016/j.forsciint.2017.07.017

Advenier, A. S., Guillard, N., Alvarez, J. C., Martrille, L., and Lorin de la Grandmaison, G. (2016). Undetermined manner of death: an autopsy series. J. Forensic Sci. 61(Suppl. 1), S154-S158.

Afshinnekoo, E., Meydan, C., Chowdhury, S., Jaroudi, D., Boyer, C., Bernstein, N., et al. (2015). Geospatial resolution of human and bacterial diversity from city-scale metagenomics. Cell Syst. 1, 72-87.

Aoyagi, M., Iwadate, K., Fukui, K., Abe, S., Sakai, K., Maebashi, K., et al. (2009). A novel method for the diagnosis of drowning by detection of Aeromonas sobria with PCR method. Leg. Med. 11, 257-259. doi: 10.1016/j.legalmed.2009.07.003

Avena, C. V., Parfrey, L. W., Leff, J. W., Archer, H. M., Frick, W. F., Langwig, K. E., et al. (2016). Deconstructing the bat skin microbiome: influences of the host and the environment. Front. Microbiol. 7:1753. doi: 10.3389/fmicb.2016.01753

Belk, A., Xu, Z. Z., Carter, D. O., Lynne, A., Bucheli, S., Knight, R., et al. (2018). Microbiome data accurately predicts the postmortem interval using random forest regression models. Genes 9, 104-128. doi: 10.3390/genes9020104

Bell, C. R., Wilkinson, J. E., Robertson, B. K., and Javan, G. T. (2018). Sexrelated differences in the thanatomicrobiome in postmortem heart samples using bacterial gene regions V1-2 and V4. Lett. Appl. Microbiol. 67, 144-153. doi: 10.1111/lam.13005

Berglund, E. C., Kiialainen, A., and Syvänen, A. C. (2011). Next-generation sequencing technologies and applications for human genetic history and forensics. Investig. Genet. 2:23. doi: 10.1186/2041-2223-2-23

Bruce, R. G., and Dettmann, M. E. (1996). Palynological analyses of Australian surface soils and their potential in forensic science. Forensic Sci. Int. 81, 77-94. doi: 10.1016/s0379-0738(96)01973-1

Bryant, V. M., and Mildenhall, D. C. (1998). Forensic palynology: a new way to catch crooks. Contrib. Ser. Am. Assoc. Stratigraph. Palynol. 33, 145-155.

Budowle, B., Schutzer, S. E., Einseln, A., Kelley, L. C., Walsh, A. C., Smith, J. A., et al. (2003). Building microbial forensics as a response to bioterrorism. Science 301, 1852-1853. doi: 10.1126/science. 1090083

Burcham, Z. M., Pechal, J. L., Schmidt, C. J., Bose, J. L., Rosch, J. W., Benbow, M. E., et al. (2019). Bacterial community succession, transmigration, and differential gene transcription in a controlled vertebrate decomposition model. Front Microbiol. 10:745. doi: 10.3389/fmicb.2019.00745

Bureau for Justice Statistics. (2019). What is the Probability of Conviction for Felony Defendants? Available online at: https://www.bjs.gov/index.cfm?ty=qa\&iid=403 (accessed February 14, 2020).

Callahan, B. J., McMurdie, P. J., and Holmes, S. P. (2017). Exact sequence variants should replace operational taxonomic units in marker-gene data analysis. ISME J. 11, 2639-2643. doi: 10.1038/ismej.2017.119

Can, I., Javan, G. T., Pozhitkov, A. E., and Noble, P. A. (2014). Distinctive thanatomicrobiome signatures found in the blood and internal organs of humans. J. Microbiol. Methods 106, 1-7. doi: 10.1016/j.mimet.2014.07.026

Capasso, L., Vento, G., Loddo, C., Tirone, C., Iavarone, F., Raimondi, F., et al. (2019). Oxidative stress and bronchopulmonary dysplasia: evidences from microbiomics, metabolomics and proteomics. Front. Pediatr. 7:30. doi: 10.3389/ fped.2019.00030

Carter, D. O., Metcalf, J. L., Bibat, A., and Knight, R. (2015). Seasonal variation of postmortem microbial communities. Forensic Sci. Med. Pathol. 11, 202-207. doi: $10.1007 / \mathrm{s} 12024-015-9667-7$

Carter, D. O., Tomberlin, J. K., Benbow, M. E., and Metcalf, J. L. (eds) (2017). Forensic Microbiology. Hoboken, NJ: John Wiley \& Sons.

Cenderadewi, M., Franklin, R. C., Peden, A. E., and Devine, S. (2019). Pattern of intentional drowning mortality: a total population retrospective cohort study in Australia, 2006-2014. BMC Public Health 19:207. doi: 10.1186/s12889-0196476-z

Chan, P. L., Yu, P. H. F., Cheng, Y. W., Chan, C. Y., and Wong, P. K. (2009). Comprehensive characterization of indoor airborne bacterial profile. J. Environ. Sci. 21, 1148-1152. doi: 10.1016/s1001-0742(08)62395-5

Chase, J., Fouquier, J., Zare, M., Sonderegger, D. L., Knight, R., Kelley, S. T., et al. (2016). Geography and location are the primary drivers of office microbiome composition. mSystems 1:e00022-16.
Christoffersen, S. (2015). The importance of microbiological testing for establishing cause of death in 42 forensic autopsies. Forensic Sci. Int. 250, 27-32. doi: $10.1016 /$ j.forsciint.2015.02.020

Clarke, T. H., Gomez, A., Singh, H., Nelson, K. E., and Brinkac, L. M. (2017). Integrating the microbiome as a resource in the forensics toolkit. Forensic Sci. Int. Genet. 30, 141-147. doi: 10.1016/j.fsigen.2017.06.008

Coil, D. A., Neches, R. Y., Lang, J. M., Jospin, G., Brown, W. E., Cavalier, D., et al. (2019). Bacterial communities associated with cell phones and shoes. PeerJ 8:e9235. doi: $10.7717 /$ peerj.9235

Daniel, R., and van Oorschot, R. A. (2011). An investigation of the presence of DNA on unused laboratory gloves. Forensic Sci. Int. 3, e45-e46.

Danko, D. C., Meleshko, D., Bezdan, D., Mason, C. E., and Hajirasouliha, I. (2019a). Minerva: an alignment and reference free approach to deconvolve linked-reads for metagenomics. Genome Res. 29, 116-124. doi: 10.1101/gr.235499.118

Danko, D. C., Bezdan, D., Afshinnekoo, E., Ahsanuddin, S., Bhattacharya, C., Butler, D. J., et al. (2019b). Global genetic cartography of urban metagenomes and anti-microbial resistance. bioRxiv [Preprint]. doi: 10.1101/724526

Daubert v. Merrell Dow Pharmaceuticals Inc (1993). 509 U.S. p. 579. Available online at: https://supreme.justia.com/cases/federal/us/509/579/ (accessed September 1, 2020).

Demanèche, S., Schauser, L., Dawson, L., Franqueville, L., and Simonet, P. (2017). Microbial soil community analyses for forensic science: application to a blind test. Forensic Sci. Int. 270, 153-158. doi: 10.1016/j.forsciint.2016.12.004

Desmond, A. U., Nicholas, O., and Emmanuel, O. O. (2018). Microbial forensics: forensic relevance of the individual person's microbial signature. Int. J. Life Sci. Scient. Res. 2455, 2037-2043.

Dobay, A., Haas, C., Fucile, G., Downey, N., Morrison, H. G., Kratzer, A., et al. (2019). Microbiome-based body fluid identification of samples exposed to indoor conditions. Forensic Sci. Int. Genet. 40, 105-113. doi: 10.1016/j.fsigen. 2019.02.010

Domínguez, A. M., Pino, J., García, A., García, I., and González, I. (2018). PW 1649 Drowning prevention campaigns, do they really work? Injury Prev. 24:A153.

Elhaik, E. (2016). a "Wear and tear" Hypothesis to explain sudden infant death syndrome. Front. Neurol. 7:180. doi: 10.3389/fneur.2016.00180

Elhaik, E. (2019). Neonatal circumcision and prematurity are associated with sudden infant death syndrome (SIDS). J. Clin. Transl. Res. 4, 136-151.

Engel, K., Sauer, J., Jünemann, S., Winkler, A., Wibberg, D., Kalinowski, J., et al. (2018). Individual-and species-specific skin microbiomes in three different estrildid finch species revealed by $16 \mathrm{~S}$ amplicon sequencing. Microb. Ecol. 76, 518-529. doi: 10.1007/s00248-017-1130-8

Erwin, P. M., Rhodes, R. G., Kiser, K. B., Keenan-Bateman, T. F., McLellan, W. A., and Pabst, D. A. (2017). High diversity and unique composition of gut microbiomes in pygmy (Kogia breviceps) and dwarf (K. sima) sperm whales. Sci. Rep. 7:7205.

Finley, S. J., Pechal, J. L., Benbow, M. E., Robertson, B. K., and Javan, G. T. (2016) Microbial signatures of cadaver gravesoil during decomposition. Microb. Ecol. 71, 524-529. doi: 10.1007/s00248-015-0725-1

Foox, J., Tighe, S. W., Nicolet, C. M., Zook, J. M., Herbert, Z. T., Warner, D., et al. (2020). Multi-platform assessment of DNA sequencing performance using human and bacterial reference genomes in the ABRF nextgeneration sequencing study. bioRxiv [Preprint]. doi: 10.1101/2020.07.23.21 8602

Franzosa, E. A., Huang, K., Meadow, J. F., Gevers, D., Lemon, K. P., Bohannan, B. J., et al. (2015). Identifying personal microbiomes using metagenomic codes. Proc. Natl. Acad. Sci. U.S.A. 112, 2930-2938.

Garrett-Bakelman, F. E., Darshi, M., Green, S. J., Gur, R. C., Lin, L., Macias, B. R., et al. (2019). The NASA Twins Study: a multidimensional analysis of a year-long human spaceflight. Science 364:eaau8650.

Goudarzi, M., Mak, T. D., Jacobs, J. P., Moon, B. H., Strawn, S. J., Braun, J., et al. (2016). An integrated multi-omic approach to assess radiation injury on the host-microbiome axis. Radiat. Res. 186, 219-234. doi: 10.1667/rr14306.1

Grantham, N. S., Reich, B. J., Laber, E. B., Pacifici, K., Dunn, R. R., Fierer, N., et al. (2019). Global forensic geolocation with deep neural networks. arXiv [Preprint]. Available online at: https://arxiv.org/abs/1905.11765 (accessed September 1, 2020).

Habtom, H., Pasternak, Z., Matan, O., Azulay, C., Gafny, R., and Jurkevitch, E. (2019). Applying microbial biogeography in soil forensics. Forensic Sci. Int. Genet. 38, 195-203. doi: 10.1016/j.fsigen.2018.11.010 
Hampton-Marcell, J. T., Lopez, J. V., and Gilbert, J. A. (2017). The human microbiome: an emerging tool in forensics. Microb. Biotechnol. 10, 228-230. doi: 10.1111/1751-7915.12699

Hanssen, E. N., Avershina, E., Rudi, K., Gill, P., and Snipen, L. (2017). Body fluid prediction from microbial patterns for forensic application. Forensic Sci. Int. Genet. 30, 10-17. doi: 10.1016/j.fsigen.2017.05.009

Huang, J., Li, Y., and Luo, Y. (2017). Bacterial community in the rumen of Tibetan sheep and Gansu alpine fine-wool sheep grazing on the QinghaiTibetan Plateau, China. J. Gen. Appl. Microbiol. 63, 122-130. doi: 10.2323/jgam. 2016.08.003

Huys, G., Coopman, V., Van Varenbergh, D., and Cordonnier, J. (2012). Selective culturing and genus-specific PCR detection for identification of Aeromonas in tissue samples to assist the medico-legal diagnosis of death by drowning. Forensic Sci. Int. 221, 11-15. doi: 10.1016/j.forsciint.2012.03.017

Ingemann-Hansen, O., Brink, O., Sabroe, S., Sørensen, V., and Charles, A. V. (2008). Legal aspects of sexual violence-does forensic evidence make a difference? Forensic Sci. Int. 180, 98-104. doi: 10.1016/j.forsciint.2008. 07.009

Innocent Project (2020). Exonerate the Innocent. Available online at: https://www. innocenceproject.org/exonerate/ (accessed September 18, 2020).

Javan, G. T., Finley, S. J., Abidin, Z., and Mulle, J. G. (2016). The thanatomicrobiome: a missing piece of the microbial puzzle of death. Front. Microbiol. 7:225. doi: 10.3389/fmicb.2016.00225

Javan, G. T., Finley, S. J., Smith, T., Miller, J., and Wilkinson, J. E. (2017). Cadaver thanatomicrobiome signatures: the ubiquitous nature of Clostridium species in human decomposition. Front. Microbiol. 8:2096. doi: 10.3389/fmicb.2017. 02096

Javan, G. T., Finley, S. J., Tuomisto, S., Hall, A., Benbow, M. E., and Mills, D. (2019). An interdisciplinary review of the thanatomicrobiome in human decomposition. Forensic Sci. Med. Pathol. 15, 75-83. doi: 10.1007/s12024-0180061-0

Jesmok, E. M., Hopkins, J. M., and Foran, D. R. (2016). Next-generation sequencing of the bacterial 16S rRNA gene for forensic soil comparison: a feasibility study. J. Forensic Sci. 61, 607-617. doi: 10.1111/1556-4029.13049

Johnson, H. R., Trinidad, D. D., Guzman, S., Khan, Z., Parziale, J. V., DeBruyn, J. M., et al. (2016). A machine learning approach for using the postmortem skin microbiome to estimate the postmortem interval. PLoS One 11:e0167370. doi: 10.1371/journal.pone.0167370

Kakizaki, E., Kozawa, S., Sakai, M., and Yukawa, N. (2009). Bioluminescent bacteria have potential as a marker of drowning in seawater: two immersed cadavers retrieved near estuaries. Leg. Med. 11, 91-96. doi: 10.1016/j.legalmed.2008. 10.004

Kaszubinski, S. F., Pechal, J. L., Smiles, K., Schmidt, C. J., Jordan, H. R., Meek, M. H., et al. (2020). Dysbiosis in the dead: human postmortem microbiome beta-dispersion as an indicator of manner and cause of death. Front. Microbiol. 11:555347. doi: 10.3389/fmicb.2020.555347

Keet, J. H., Ellis, A. G., Hui, C., and Le Roux, J. J. (2019). Strong spatial and temporal turnover of soil bacterial communities in South Africa's hyperdiverse fynbos biome. Soil Biol. Biochem. 136:107541. doi: 10.1016/j.soilbio.2019. 107541

Kodama, W. A., Xu, Z., Metcalf, J. L., Song, S. J., Harrison, N., Knight, R., et al. (2019). Trace evidence potential in postmortem skin microbiomes: from death scene to morgue. J. Forensic Sci. 64, 791-798. doi: 10.1111/1556-4029.13949

Kõljalg, S., Mändar, R., Sõber, T., Rööp, T., and Mändar, R. (2017). High level bacterial contamination of secondary school students' mobile phones. Germs 7, 73-77. doi: 10.18683/germs.2017.1111

Komaroff, A. L. (2018). The microbiome and risk for atherosclerosis. JAMA 319, 2381-2382. doi: 10.1001/jama.2018.5240

Koroglu, M., Gunal, S., Yildiz, F., Savas, M., Ozer, A., and Altindis, M. (2015). Comparison of keypads and touch-screen mobile phones/devices as potential risk for microbial contamination. J. Infect. Dev. Ctries. 9, 1308-1314. doi: $10.3855 /$ jidc. 6171

Koscova, J., Hurnikova, Z., and Pistl, J. (2018). Degree of bacterial contamination of mobile phone and computer keyboard surfaces and efficacy of disinfection with chlorhexidine digluconate and triclosan to its reduction. Int. J. Environ. Res. Public Health 15:2238. doi: 10.3390/ijerph15102238

Kuiper, I. (2016). Microbial forensics: next-generation sequencing as catalyst. EMBO Rep. 17, 1085-1087. doi: 10.15252/embr.201642794
Kurli, R., Chaudhari, D., Pansare, A. N., Khairnar, M., Shouche, Y. S., and Rahi, P. (2018). Cultivable microbial diversity associated with cellular phones. Front. Microbiol. 9:1229. doi: 10.3389/fmicb.2018.01229

LaPorte, G. (2017). Wrongful convictions and DNA exonerations: Understanding the role of forensic science. NIJ J. 279:250705.

Lax, S., Hampton-Marcell, J. T., Gibbons, S. M., Colares, G. B., Smith, D., Eisen, J. A., et al. (2015). Forensic analysis of the microbiome of phones and shoes. Microbiome 3:21.

Lax, S., Smith, D. P., Hampton-Marcell, J., Owens, S. M., Handley, K. M., Scott, N. M., et al. (2014). Longitudinal analysis of microbial interaction between humans and the indoor environment. Science 345, 1048-1052. doi: 10.1126/ science. 1254529

Lee, S. Y., Woo, S. K., Lee, S. M., Ha, E. J., Lim, K. H., Choi, K. H., et al (2017). Microbiota composition and pulmonary surfactant protein expression as markers of death by drowning. J. Forensic Sci. 62, 1080-1088. doi: 10.1111/ 1556-4029.13347

Lucci, A., Campobasso, C. P., Cirnelli, A., and Lorenzini, G. (2008). A promising microbiological test for the diagnosis of drowning. Forensic Sci. Int. 182, 20-26. doi: 10.1016/j.forsciint.2008. 09.004

Lucci, A., and Cirnelli, A. (2007). A microbiological test for the diagnosis of death by drowning. Forensic Sci. Int. 168, 34-36. doi: 10.1016/j.forsciint.2006. 06.050

Luongo, J. C., Barberán, A., Hacker-Cary, R., Morgan, E. E., Miller, S. L., and Fierer, N. (2017). Microbial analyses of airborne dust collected from dormitory rooms predict the sex of occupants. Indoor Air 27, 338-344. doi: 10.1111/ina.12302

Lutz, H., Vangelatos, A., Gottel, N., Speed, E., Osculati, A., Visona, S., et al. (2019). Manner of death and demographic effects on microbial community composition in organs of the human cadaver. bioRxiv [Preprint]. doi: 10.1101/ 752576

MacCallum, W. G., and Hastings, T. W. (1899). A case of acute endocarditis caused by Micrococcus zymogenes (nov. spec.), with a description of the microorganism. J. Exp. Med. 4, 521-534. doi: 10.1084/jem.4.5-6.521

Macdonald, C. A., Ang, R., Cordiner, S. J., and Horswell, J. (2011). Discrimination of soils at regional and local levels using bacterial and fungal T-RFLP profiling. J. Forensic Sci. 56, 61-69. doi: 10.1111/j.1556-4029.2010.01542.x

Marella, G. L., Feola, A., Marsella, L. T., Mauriello, S., Giugliano, P., and Arcudi, G. (2019). Diagnosis of drowning, an everlasting challenge in forensic medicine: review of the literature and proposal of a diagnostic algorithm. Acta Med. 35, 900-919.

Mayuoni-Kirshenbaum, L., Waiskopf, O., Finkelstein, N., and Pasternak, Z. (2020). How did the DNA of a suspect get to the crime scene? A practical study in DNA transfer during lock-picking. Aust. J. Forensic Sci. 52, 1-11. doi: 10.1080/ 00450618.2020.1793384

McIntyre, A. B. R., Alexander, N., Grigorev, K., Bezdan, D., Sichtig, H., Chiu, C. Y., et al. (2019). Single-molecule sequencing detection of N6-methyladenine in microbial reference materials. Nat. Commun. 10:579.

McIntyre, A. B. R., Ounit, R., Afshinnekoo, E., Prill, R. J., Hénaff, E., Alexander, N., et al. (2017). Comprehensive benchmarking and ensemble approaches for metagenomic classifiers. Genome Biol. 18:182.

McKenzie, V. J., Bowers, R. M., Fierer, N., Knight, R., and Lauber, C. L. (2012). Cohabiting amphibian species harbor unique skin bacterial communities in wild populations. ISME J. 6, 588-596. doi: 10.1038/ismej.2011.129

Meadow, J. F., Altrichter, A. E., and Green, J. L. (2014). Mobile phones carry the personal microbiome of their owners. PeerJ 2:e447. doi: 10.7717/peerj.447

MetaSUB International Consortium (2016). The metagenomics and metadesign of the subways and urban biomes (MetaSUB) international consortium inaugural meeting report. Microbiome 4:24.

Metcalf, J. L., Parfrey, L. W., Gonzalez, A., Lauber, C. L., Knights, D., Ackermann, G., et al. (2013). A microbial clock provides an accurate estimate of the postmortem interval in a mouse model system. eLife 2:e01104.

Metcalf, J. L., Xu, Z. Z., Bouslimani, A., Dorrestein, P., Carter, D. O., and Knight, R. (2017). Microbiome tools for forensic science. Trends Biotechnol. 35, 814-823. doi: 10.1016/j.tibtech.2017.03.006

Morgan, R. M., and Levin, E. A. (2019). A crisis for the future of forensic science: lessons from the UK of the importance of epistemology for funding research and development. Forensic Sci. Int. 1, 243-252. doi: 10.1016/j.fsisyn.2019.09. 002 
Neckovic, A., van Oorschot, R. A., Szkuta, B., and Durdle, A. (2020). Investigation of direct and indirect transfer of microbiomes between individuals. Forensic Sci. Int. Genet. 45:102212. doi: 10.1016/j.fsigen.2019.102212

Noel, S., Martina-Lingua, M. N., Bandapalle, S., Pluznick, J., Hamad, A. R. A., Peterson, D. A., et al. (2014). Intestinal microbiota-kidney cross talk in acute kidney injury and chronic kidney disease. Nephron Clin. Pract. 127, 139-143. doi: $10.1159 / 000363209$

Office for National Statistics (2019). Homicide in England and Wales: Year Ending March 2019. Available online at: https://www.ons. gov.uk/peoplepopulationandcommunity/crimeandjustice/articles/ homicideinenglandandwales/yearendingmarch2019 (accessed February 14, 2020).

Ogilvie, L. A., and Jones, B. V. (2015). The human gut virome: a multifaceted majority. Front. Microbiol. 6:918. doi: 10.3389/fmicb.2015.00918

Oliveira, M., and Amorim, A. (2018). Microbial forensics: new breakthroughs and future prospects. Appl. Microbiol. Biotechnol. 102, 10377-10391. doi: 10.1007/ s00253-018-9414-6

Pasternak, Z., Al-Ashhab, A., Gatica, J., Gafny, R., Avraham, S., Minz, D., et al. (2013). Spatial and temporal biogeography of soil microbial communities in arid and semiarid regions. PLoS One 8:e69705. doi: 10.1371/journal.pone. 0069705

Pasternak, Z., Luchibia, A. O., Matan, O., Dawson, L., Gafny, R., Shpitzen, M., et al. (2019). Mitigating temporal mismatches in forensic soil microbial profiles. Aust. J. Forensic Sci. 51, 685-694. doi: 10.1080/00450618.2018.14 50897

Pechal, J. L., Crippen, T. L., Benbow, M. E., Tarone, A. M., Dowd, S., and Tomberlin, J. K. (2014). The potential use of bacterial community succession in forensics as described by high throughput metagenomic sequencing. Int. J. Legal Med. 128, 193-205. doi: 10.1007/s00414-013-0872-1

Pechal, J. L., Schmidt, C. J., Jordan, H. R., and Benbow, M. E. (2018). A largescale survey of the postmortem human microbiome, and its potential to provide insight into the living health condition. Sci. Rep. 8:5724.

Phan, K., Barash, M., Spindler, X., Gunn, P., and Roux, C. (2020). Retrieving forensic information about the donor through bacterial profiling. Int. J. Legal Med. 134, 21-29. doi: 10.1007/s00414-019-02069-2

President's Council of Advisors on Science and Technology (US) (2016). Report to the President. Forensic Science in Criminal Courts: Ensuring scientific validity of Feature-Comparison Methods. Washington, DC: Executive Office of the President of the United States.

Rambaud, C., Guibert, M., Briand, E., Grangeot-Keros, L., Coulomb-L'Herminé, A., and Dehan, M. (1999). Microbiology in sudden infant death syndrome (SIDS) and other childhood deaths. FEMS Immunol. Med. Microbiol. 25, 59-66. doi: 10.1111/j.1574-695x.1999.tb01327.x

Richardson, M., Gottel, N., Gilbert, J. A., and Lax, S. (2019). Microbial similarity between students in a common dormitory environment reveals the forensic potential of individual microbial signatures. mBio 10:e1054-19.

Rosenfeld, J., Reeves, D., Brugler, M. R., Narechania, A., Simon, S., Kolokotronis, S., et al. (2016). Genome assembly, annotation, and urban phylogenomics of the bedbug (Cimex lectularius). Nat. Commun. 7:10164.

Russo, C. D., Weller, D. W., Nelson, K. E., Chivers, S. J., Torralba, M., and Grimes, D. J. (2018). Bacterial species identified on the skin of bottlenose dolphins off southern California via next generation sequencing techniques. Microb. Ecol. 75, 303-309. doi: 10.1007/s00248-017-1071-2

Rutty, G. N., Bradley, C. J., Biggs, M. J., Hollingbury, F. E., Hamilton, S. J., Malcomson, R. D., et al. (2015). Detection of bacterioplankton using PCR probes as a diagnostic indicator for drowning; the Leicester experience. Leg. Med. 17, 401-408. doi: 10.1016/j.legalmed.2015.06.001

Ryan, F. J. (2019). Application of machine learning techniques for creating urban microbial fingerprints. Biol. Direct 14:13.

Salzmann, A. P., Russo, G., Aluri, S., and Haas, C. (2019). Transcription and microbial profiling of body fluids using a massively parallel sequencing approach. Forensic Sci. Int. Genet. 43:102149. doi: 10.1016/j.fsigen.2019.10 2149

Sanachai, A., Katekeaw, S., and Lomthaisong, K. (2016). Forensic soil investigation from the $16 \mathrm{~S}$ rDNA profiles of soil bacteria obtained by denaturing gradient gel electrophoresis. Chiang Mai J. Sci. 43, 748-755.

Sangero, B., and Halpert, M. (2007). Why a conviction should not be based on a single piece of evidence: a proposal for reform. Jurimetrics $48,43-94$.
Schmedes, S. E., Woerner, A. E., and Budowle, B. (2017). Forensic human identification using skin microbiomes. Appl. Environ. Microbiol. 83:e01672-17.

Schmedes, S. E., Woerner, A. E., Novroski, N. M., Wendt, F. R., King, J. L., Stephens, K. M., et al. (2018). Targeted sequencing of clade-specific markers from skin microbiomes for forensic human identification. Forensic Sci. Int. Genet. 32, 50-61. doi: 10.1016/j.fsigen.2017.10.004

Sender, R., Fuchs, S., and Milo, R. (2016). Revised estimates for the number of human and bacteria cells in the body. PLoS Biol. 14:e1002533. doi: 10.1371/ journal.pbio. 1002533

Shamarina, D., Stoyantcheva, I., Mason, C. E., Bibby, K., and Elhaik, E. (2017). Communicating the promise, risks, and ethics of large-scale, open space microbiome and metagenome research. Microbiome 5, 132-141.

Singh, B., Minick, K. J., Strickland, M. S., Wickings, K. G., Crippen, T. L., Tarone, A. M., et al. (2018). Temporal and spatial impact of human cadaver decomposition on soil bacterial and arthropod community structure and function. Front. Microbiol. 8:2616. doi: 10.3389/fmicb.2017.02616

Smith, T. F., and Waterman, M. S. (1992). The continuing case of the Florida dentist. Science 256, 1155-1156. doi: 10.1126/science.256.5060.1155

Song, S. J., Lauber, C., Costello, E. K., Lozupone, C. A., Humphrey, G., Berg-Lyons, D., et al. (2013). Cohabiting family members share microbiota with one another and with their dogs. eLife 2:e00458.

Statnikov, A., Henaff, M., Narendra, V., Konganti, K., Li, Z., Yang, L., et al. (2013). A comprehensive evaluation of multicategory classification methods for microbiomic data. Microbiome 1:11.

Szydlowski, L., Skierska, A., Markiewicz-Loskot, G., Mazurek, B., Morka, A., and Undas, A. (2013). The role of Interleukin-6, its- $174 \mathrm{G}>\mathrm{C}$ polymorphism and C-reactive protein in idiopathic cardiac arrhythmias in children. Adv. Med. Sci. 58, 320-325. doi: 10.2478/ams-2013-0003

Torres, S., Clayton, J. B., Danzeisen, J. L., Ward, T., Huang, H., Knights, D., et al. (2017). Diverse bacterial communities exist on canine skin and are impacted by cohabitation and time. PeerJ 5:e3075. doi: 10.7717/peerj.3075

Tridico, S. R., Murray, D. C., Addison, J., Kirkbride, K. P., and Bunce, M. (2014). Metagenomic analyses of bacteria on human hairs: a qualitative assessment for applications in forensic science. Investig. Genet. 5:16.

Uchiyama, T., Kakizaki, E., Kozawa, S., Nishida, S., Imamura, N., and Yukawa, N. (2012). A new molecular approach to help conclude drowning as a cause of death: simultaneous detection of eight bacterioplankton species using real-time PCR assays with TaqMan probes. Forensic Sci. Int. 222, 11-26. doi: 10.1016/j. forsciint.2012.04.029

van Belkum, A. (1994). DNA fingerprinting of medically important microorganisms by use of PCR. Clin. Microbiol. Rev. 7, 174-184. doi: $10.1128 / \mathrm{cmr} .7 .2 .174-184.1994$

Vázquez-Baeza, Y., Callewaert, C., Debelius, J., Hyde, E., Marotz, C., Morton, J. T., et al. (2018). Impacts of the human gut microbiome on therapeutics. Annu. Rev. Pharmacol. Toxicol. 58, 253-270.

Voloshynovych, V. M., Kasala, R. O., Stambulska, U. Y., and Voloshynovych, M. S. (2019). Determination the presence of amplification products of $16 \mathrm{~s}$ rRNA microcystis aeruginosa as a biomarker of drowning. Rom. J. Leg. Med. 27, 16-21. doi: $10.4323 /$ rjlm.2019.16

Walker, A. R., and Datta, S. (2019). Identification of city specific important bacterial signature for the MetaSUB CAMDA challenge microbiome data. Biol. Direct 14:11.

Walsh, K., Hussemann, J., Flynn, A., Yahner, J., and Golian, L. (2017). Estimating the Prevalence of Wrongful Convictions. Final Report 251115. (Washington, DC: Urban Institute), 1-13.

Wang, S., Song, F., Wang, Y., Huang, Y., Xie, B., and Luo, H. (2019). High resolution melting analysis (HRM) based on 16SrRNA as a tool for personal identification with the human oral microbiome. Forensic Sci. Int. Genet. 7, 161-163. doi: 10.1016/j.fsigss.2019.09.063

Watanabe, H., Nakamura, I., Mizutani, S., Kurokawa, Y., Mori, H., Kurokawa, K., et al. (2018). Minor taxa in human skin microbiome contribute to the personal identification. PLoS One 13:e0199947. doi: 10.1371/journal.pone.0199947

Williams, D. W., and Gibson, G. (2017). Individualization of pubic hair bacterial communities and the effects of storage time and temperature. Forensic Sci. Int. Genet. 26, 12-20. doi: 10.1016/j.fsigen.2016.09.006

Willis, S. M., McKenna, L., McDermott, S., O’Donell, G., Barrett, A., Rasmusson, B., et al. (2015). ENFSI Guideline for Evaluative Reporting in Forensic Science. Wiesbaden: European Network of Forensic Science Institutes. 
Woerner, A. E., Novroski, N. M., Wendt, F. R., Ambers, A., Wiley, R., Schmedes, S. E., et al. (2019). Forensic human identification with targeted microbiome markers using nearest neighbor classification. Forensic Sci. Int. Genet. 38, 130-139. doi: 10.1016/j.fsigen.2018.10.003

Young, J. M., Austin, J. J., and Weyrich, L. S. (2017). Soil DNA metabarcoding and high-throughput sequencing as a forensic tool: considerations, potential limitations and recommendations. FEMS Microbiol. Ecol. 93:fiw207. doi: 10. 1093/femsec/fiw207

Young, J. M., Weyrich, L. S., Breen, J., Macdonald, L. M., and Cooper, A. (2015). Predicting the origin of soil evidence: high throughput eukaryote sequencing and MIR spectroscopy applied to a crime scene scenario. Forensic Sci. Int. 251, 22-31. doi: 10.1016/j.forsciint.2015.03.008

Zeng, B., Zhao, J., Guo, W., Zhang, S., Hua, Y., Tang, J., et al. (2017). High-altitude living shapes the skin microbiome in humans and pigs. Front. Microbiol. 8:1929. doi: 10.3389/fmicb.2017.01929

Zhang, Y., Pechal, J. L., Schmidt, C. J., Jordan, H. R., Wang, W. W., Benbow, M. E., et al. (2019). Machine learning performance in a microbial molecular autopsy context: a cross-sectional postmortem human population study. PLoS One 14:e0213829. doi: 10.1371/journal.pone.0213829

Zhao, J., Yao, Y., Li, D., Xu, H., Wu, J., Wen, A., et al. (2018). Characterization of the gut microbiota in six geographical populations of Chinese Rhesus macaques (Macaca mulatta), implying an adaptation to high-altitude environment. Microb. Ecol. 76, 565-577. doi: 10.1007/s00248-0181146-8

Zhou, W., and Bian, Y. (2018). Thanatomicrobiome composition profiling as a tool for forensic investigation. Forensic Sci. Res. 3, 105-110. doi: 10.1080/20961790. 2018.1466430

Zhou, X., Jiang, X., Yang, C., Ma, B., Lei, C., Xu, C., et al. (2016). Cecal microbiota of Tibetan Chickens from five geographic regions were determined by $16 \mathrm{~S}$ rRNA sequencing. Microbiologyopen 5, 753-762. doi: 10.1002/mbo3.367

Conflict of Interest: EE consults the DNA Diagnostics Center. CM is a co-founder of Biotia, Inc.

The remaining authors declare that the research was conducted in the absence of any commercial or financial relationships that could be construed as a potential conflict of interest.

Copyright (C) 2021 Robinson, Pasternak, Mason and Elhaik. This is an open-access article distributed under the terms of the Creative Commons Attribution License (CC BY). The use, distribution or reproduction in other forums is permitted, provided the original author(s) and the copyright owner(s) are credited and that the original publication in this journal is cited, in accordance with accepted academic practice. No use, distribution or reproduction is permitted which does not comply with these terms. 